\title{
A Pilot Investigation of the Impact of Cognitive Demand on Turn Signal Use during Lane Changes in Actual Highway Conditions across Multiple Age Groups
}

\author{
Birsen Donmez ${ }^{1,2}$, Bryan Reimer ${ }^{2}$, Bruce Mehler ${ }^{2}$, Martin Lavallière ${ }^{2,3}$ \& Joseph F. Coughlin ${ }^{2}$ \\ ${ }^{1}$ University of Toronto, Department of Mechanical and Industrial Engineering, Toronto, Canada \\ ${ }^{2}$ MIT AgeLab \& New England University Transportation Center, AgeLab, Cambridge, MA, USA \\ ${ }^{3}$ Université Laval, Faculty of Medicine, \& Vieillissement, Centre de recherche FRSQ du Centre \\ hospitalier affilié universitaire de Québec, Québec, Canada
}

\begin{abstract}
In a field study, we examined turn signal use characteristics during highway-lane changes across three age groups (20-29, 40-49, and 60-69) and under varying levels of secondary cognitive demand. Secondary cognitive demand decreased the likelihood of turn signal use, and delayed the onset and turnoff of the turn signals. These results suggest that added cognitive load can lead to a neglect of operational tasks in face of competition over limited resources. No major effects of age or relative level of secondary cognitive demand were observed.
\end{abstract}

\section{INTRODUCTION}

Lane changing is a driving maneuver frequently associated with accidents (Pande \& Abdel-Aty, 2006). Turn signal use during a lane change communicates a driver's intention to perform a lane change to other drivers. The failure of turn signal use is a risky behavior that can lead to miscommunications between drivers. This failure also interferes with the effectiveness of lane departure warning systems, which alert drivers if they are drifting off their lane without their turn signal on.

In a field study, Boyce and Geller (2002) found that younger drivers (18-25 years old) used their turn signals approximately $90 \%$ of the time, whereas middle-aged (35-45) and older $(65+)$ drivers signalled less than $80 \%$ of the time. The differences in these rates did not reach statistical significance. Without consideration of age effects, Kiefer and Hankey (2008) observed that drivers in a field study failed about $23 \%$ of the time to engage their turn signals before the initiation of a lane change.

From the 100-car naturalistic study, Fitch et al. (2009) reported turn signal use in planned lane changing events that were classified as involving a driver (subject vehicle) nearly sideswiping another vehicle. Of the 26 events where drivers were making left-lane changes, turn signal was used in 22 (85\%). Of the 22 drivers who used their turn signals, 14 drivers (64\%) initiated their turn signals prior to starting the lane change. In right-lane-change events, 18 of 25 drivers (72\%) used their turn signals; eight of these drivers (44\%) initiated their turn signals before starting the lane change. In another naturalistic study conducted with 16 drivers from Virginia, the signal use rates were much lower: $48 \%$ for left lane changes and 35\% for right lane changes with significant variability across drivers with a range of $0 \%$ to $92 \%$ (Lee, Olsen, \& Wierwille, 2004). Research also suggests that there is variability as to when the turn signal is engaged with respect to the initiation of a lane change maneuver (Hetrick, 1997). An earlier turn signal onset can be considered to be a safer behavior than a delayed one, with the potential of providing other drivers more time to react to an imminent lane change.
Humans are generally considered to have finite information processing resources (Wickens, 1984; Wickens \& McCarley, 2008), and situations that make multiple calls on these resources, particularly those that require divided attention, may tax capacity to the point that performance and safety margins suffer. To the best of our knowledge, no previous research has examined the effects of added cognitive demand on turn signal use.

By examining turn signal use, this paper expands upon recently published work on lane change behavior, physiological reactivity, and changes in visual behavior in response to graded levels of a secondary cognitive demand across different age groups, 20-29, 40-49, and 60-69 years old (Lavalliere et al., 2011; Mehler, Reimer, \& Coughlin, 2010; Reimer, Mehler, Wang, \& Coughlin, 2010). The data were captured during an extended period of driving on a multi-lane interstate during which participants were free to maintain or adjust their lane positioning at will. Analysis was limited to cases that were classified as being largely unaffected by traffic flow (e.g., cases with extended periods of stop and go traffic were excluded) and adverse weather conditions. The study was conducted during daytime and non-rush hour periods. The availability of this dataset provided an opportunity to examine turn signal use of different age groups during lane changes under both single task driving and under conditions of objectively defined levels of cognitive secondary task load.

Findings on lane change and choice behavior were published in Lavalliere et al. (2011). In general, older adults were found to adopt a more conservative driving style by not traveling in the leftmost lane as much as the younger groups and being less likely to change lanes than drivers in their 40's. Regardless of demand level, cognitive load reduced the likelihood of lane changes for all age groups, suggesting a tendency in drivers to regulate their behavior in a risk reducing direction in response to added cognitive demand. The current paper analyzes turn signal use characteristics during lane changes observed in this field study. 


\section{METHOD}

As noted above, the signal use data presented here are drawn from a dataset collected as part of a larger project. An overview of subject selection and methods is provided below; for additional detail see (Mehler, et al., 2010; Reimer, et al., 2010).

\section{Participants}

The sample considered here consisted of 106 individuals and was balanced by gender and across three age groups 20-29 $(n=36), 40-49(n=35)$, and 60-69 $(n=35)$. The average age by group was 24.6 (SD 2.7), 44.4 (SD 3.0), and 63.3 (SD 3.1). The male and female participants did not differ significantly by age within each group $(F(1,34)=.86, p=.36 ; F(1,33)=.83$, $\mathrm{p}=.37 ; \mathrm{F}(1,33)=.22, \mathrm{p}=.64)$. Participants were experienced drivers, driving more than three times a week and having held a valid driver's license for over three years. They were also required to be free of accidents for the past year. The participant group was considered to be relatively healthy compared to an unscreened community sample based on self report and specified exclusion criteria. Compensation of $\$ 60$ was provided for the 3-hour experiment.

\section{Apparatus and Secondary Task Demand}

Participants drove an instrumented Volvo XC 90 equipped for time synchronized data collection. Data presented were recorded from the vehicle's CAN bus and a camera mounted near the center of the vehicle facing forward.

Three levels of an auditory delayed recall task (n-back) were employed to increase drivers' workload. Each level consisted of four 30-second trials during which 10 single digit numbers $(0-9)$ were presented randomly at a spacing of 2.25 seconds. At the lowest level of demand (0-back), drivers were to repeat verbally each digit as it was presented. At the moderate level of demand (1-back), drivers were to respond with the next to last number presented. At the highest level of demand (2-back), the number two places back in the sequence was to be repeated. The form of this task was used in earlier studies (e.g., Mehler, Reimer, Coughlin, \& Dusek, 2009; Reimer, 2009) and was based on recommendations by Zeitlin (1993) (for additional details on the delayed digit recall task see Mehler, Reimer, \& Dusek, 2011). The secondary task period was initiated approximately at the same geographical location for each driver.

As detailed in Lavalliere et al. (2011), secondary task performance for the current sample in the relatively low demand 0-back condition was near perfect across all three age groups (M 99.9\%; SD 0.90). Performance dropped progressively with demand level for the more challenging 1back (M 95.4\%; SD 7.65) and 2-back (M 87.2\%; SD 15.42) conditions. A review of individual performance scores showed that all participants remained engaged in attempting to perform at all three difficulty levels. Heart rate and skin conductance level (Mehler, et al., 2010) increased significantly with each level of demand relative to single task driving across the age groups, providing additional substantiation that overall cognitive demand / workload was elevated during the secondary task periods. As detailed in Reimer et al. (2010), changes in visual attention and driving speed were found as well. With added demand, gaze allocation became centralized and drivers adopted a somewhat slower and more conservative driving speed.

\section{Procedure}

Participants signed an approved informed consent and completed a questionnaire covering driving and health history. They were then extensively trained on the secondary task prior to beginning the active driving portion of the experiment. A research associate, seated in the back of the car, operated the data collection equipment, provided driving directions, and monitored the participants to ensure that they had adequate control of the vehicle at all times. Approximately 30 minutes of driving were provided for habituation prior to the study period. As indicated in Table 1, the study period consisted of an initial six minutes of single task driving followed by the three levels of the n-back task. Each task was presented over a two-minute interval (four 30-second trials) and each task was followed by 2 minutes of single task driving. The presentation order for the three levels of the task was counterbalanced across the sample. Pre-recorded instructions introduced each task, informing participants of the form of response to use for the current task period ( 0,1 , or 2-back). These instruction periods were 18 seconds in duration and were not included in the analysis.

Table 1. Data analysis periods

\begin{tabular}{lrl}
\hline Period & $\begin{array}{r}\text { Duration } \\
\text { (min:sec) }\end{array}$ & Content \\
\hline $\begin{array}{l}\text { Habituation } \\
\text { Pre-task }\end{array}$ & $\sim 30: 00$ & $\begin{array}{l}\text { Single task driving } \\
\text { Single task driving } \\
\text { (2-minute reference period: }\end{array}$ \\
& & $\begin{array}{l}\text { min. 3:30 to min. 5:30) } \\
\text { Dual task - Four 10 item trials }\end{array}$ \\
$1^{\text {st }}$ task & $2: 00$ & $\begin{array}{l}\text { Single task driving } \\
\text { Recovery }\end{array}$ \\
$2^{\text {nd }}$ task & $2: 00$ & Dual task - Four 10 item trials \\
Recovery & $2: 00$ & Single task driving \\
$3^{\text {rd }}$ task & $2: 00$ & Dual task - Four 10 item trials \\
Recovery & $2: 00$ & Single task driving \\
\hline
\end{tabular}

The experiment was conducted on Interstate 93 traveling north from Boston, Massachusetts, and the area of data collection was consistent for all participants. The posted speed limit was $104.6 \mathrm{~km} / \mathrm{h}$ (65 mph). When entering the highway, participants were prompted: "We are going to be driving north on 93 for approximately 40 minutes. You can continue driving in this lane or move into another lane so that you are comfortable with the traffic flow." Participants were thus allowed to select a lane of travel and pass other vehicles at will. There were between two and four travel lanes across the portion of highway used in the study. The distribution of the number of travel lanes across the sample was not uniform due to the nature of variations in traffic and driver speed that affected the start of the data assessment period. 


\section{Data Coding and Data Periods used in the Analysis}

Lane changes were identified through a manual analysis of video recordings using Microsoft Media Player at a resolution of $1 \mathrm{~Hz}$. Turn signal use was identified from time synchronized data recorded at $10 \mathrm{~Hz}$ from the vehicle's CAN bus. The procedure for classification of lane changes was analogous to Olsen et al. (2002) and consistent with Cooper et al. (2009). The onset of each lane change was classified as the point when the vehicle was observed to be first moving in a lateral direction toward the destination lane. The completion of each lane change was recorded as the point where the vehicle was fully centered in the destination lane. A lane crossing was classified as when the middle of the car crossed the lane marker. Only lane changes that resulted in the centerline of the vehicle crossing over the dividing line were considered; partial motions toward an adjacent lane were not coded. A lane change was assigned to the period in which it was initiated. A single analyst performed the data coding; selected cases were verified by the $4^{\text {th }}$ author.

Three data periods were analyzed: pre-task, n-back (cognitive demand tasks), and recovery. The pre-task period consisted of six minutes of single task driving prior to the initiation of the first n-back task. The n-back period consisted of the six minutes of dual task data corresponding to the aggregate of the three separate two-minute-long secondary tasks. The six minutes of data for the recovery period was drawn from the two minutes of single task driving that followed each of the three dual task periods. Also considered was an analysis of each of the three task demand levels $(0,1$, and 2-back). Since each task was two minutes long, a twominute reference period was used for comparison to the three task demand levels. To maintain consistency with Mehler et al. (2010) and Reimer et al. (2010), the two-minute reference period was selected as minutes 3.5 to 5.5 of the pre-task period. Gender was initially included in all statistical analysis, but was later dropped from the final models, as it was not a significant predictor in any of the models.

\section{RESULTS}

\section{Turn Signal Use}

Table 2 presents the rate of turn signal use during lane changes. A logistic regression model was developed to predict the odds of turn signal use across different age groups for three study periods: the six-minute period prior to the admission of the n-back tasks (pre-task), the aggregate of the three two-minute-long n-back tasks (n-back), and the combination of the three two-minute intervals of single task driving following each n-back task (recovery). Each of these three periods was six-minutes in duration. Repeated measures were accounted for by using Generalized Estimating Equations (GEE).

Odds of turn signal use is defined as the probability of the turn signal being used divided by the probability of it not being used. A higher value of odds indicates a higher likelihood of turn signal use. Moreover, if the value of odds is larger than one, then the probability of observing a signal use is greater than $50 \%$.

Table 2. Rate of turn signal use by age and study period (pretask, n-back, and recovery)

\begin{tabular}{llll}
\hline Age & $\begin{array}{l}\text { Study } \\
\text { period }\end{array}$ & $\begin{array}{l}\text { Rate of } \\
\text { signal use (\%) }\end{array}$ & $\begin{array}{l}\text { Total number } \\
\text { of lane changes }\end{array}$ \\
\hline \multirow{2}{*}{ 20’s } & Pre-task & 87 & 68 \\
$(\mathrm{n}=36)$ & N-back & 80 & 55 \\
& Recovery & 86 & 95 \\
\hline \multirow{2}{*}{ 40’s } & Pre-task & 77 & 78 \\
$(\mathrm{n}=35)$ & N-back & 86 & 65 \\
& Recovery & 93 & 84 \\
\hline \multirow{2}{*}{ 60’s } & Pre-task & 93 & 59 \\
$(\mathrm{n}=35)$ & N-back & 83 & 30 \\
& Recovery & 96 & 71 \\
\hline
\end{tabular}

Neither age, nor the study period $x$ age interaction was significant ( $\mathrm{p}>.05)$. A significant effect was observed for study period $\left(\chi^{2}(2)=6.66, p=.04\right)$. The odds of turn signal use were highest during the recovery period (12.3), followed by the pretask period (6.7), with the lowest odds for the n-back period (5.0). In terms of likelihood, the participants were most likely to use their turn signals in the recovery period, followed by the pre-task period, and then the n-back period. Only, the difference between the recovery and the n-back periods was statistically significant. The odds of turn signal use during the recovery period were $146 \%$ (95\% CI: 22\%, 395\%) higher than the odds of turn signal use during the n-back period.

Table 3. Rate of turn signal use by age and demand level (reference, 0, 1, and 2-back task)

\begin{tabular}{llll}
\hline Age & $\begin{array}{l}\text { Demand } \\
\text { level }\end{array}$ & $\begin{array}{l}\text { Rate of } \\
\text { signal use (\%) }\end{array}$ & $\begin{array}{l}\text { Total number of } \\
\text { lane changes }\end{array}$ \\
\hline \multirow{2}{*}{ 20's } & Reference & 89 & 27 \\
$(\mathrm{n}=36)$ & Low & 84 & 25 \\
& Moderate & 73 & 15 \\
& High & 80 & 15 \\
\hline \multirow{2}{*}{ 40’s } & Reference & 73 & 26 \\
$(\mathrm{n}=35)$ & Low & 87 & 23 \\
& Moderate & 89 & 28 \\
& High & 79 & 14 \\
\hline \multirow{2}{*}{ 60’s } & Reference & 94 & 18 \\
$(\mathrm{n}=35)$ & Low & 80 & 10 \\
& Moderate & 82 & 11 \\
& High & 89 & 9 \\
\hline
\end{tabular}

A finer break down of the secondary task period is provided in Table 3 . These data are based on the two-minute windows represented by the three separate cognitive difficulty levels (low, moderate, and high) and where "reference" represents two-minutes prior to the first dual task period. Another logistic regression model was built to analyze these data. Neither age, nor demand level was significant ( $>>05$ ). Fewer number of observations during the 2-min periods may have led to a loss in statistical power. 


\section{Turn Signal Onset Time}

Figure 1 presents the turn signal onset times with respect to the initiation of lane change maneuvers. A positive value indicates that the turn signal was onset before the start of the lane change whereas a negative value indicates that the turn signal was onset after the initiation of the lane change. These data do not include cases when the turn signal was not used during a lane change. As can be seen from Figure 1, in which the median value is indicated with a thick solid horizontal line, during pre-task and recovery periods, the onset of the turn signal before the initiation of the lane change was about as equally likely as the onset of the turn signal after the initiation of the lane change. The mean onset times were close to zero for pre-task ( $0.03 \mathrm{sec})$ and recovery $(0.07 \mathrm{sec})$ periods, indicating that the participants, on the average, engaged their turn signals around the initiation of the lane change. In contrast, the mean onset time was negative for the n-back period $(-0.87 \mathrm{sec})$, suggesting that with cognitive demand, the participants, on the average, engaged their turn signals after the initiation of the lane change.

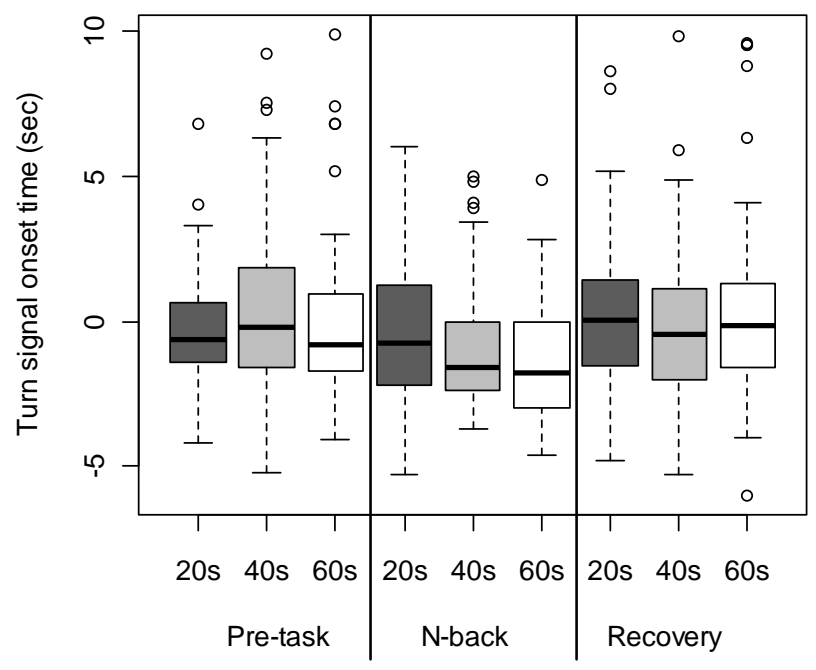

Figure 1. Turn signal onset time (sec) for each age and study period (pre-task, n-back, and recovery)

A mixed linear model was used for the statistical analysis. Neither age, nor the study period $x$ age interaction was significant ( $\mathrm{p}>$.05). A significant effect was observed for study period $(\mathrm{F}(2,499)=8.11, \mathrm{p}=.0003)$. Given that the participants used their turn signal, they onset the signal earlier in the pretask and recovery periods compared to the n-back task period (pre-task vs. n-back: $1.06 \mathrm{sec}, \mathrm{t}(494)=3.60, \mathrm{p}=.0003$; recovery vs. n-back: $1.03 \mathrm{sec}, \mathrm{t}(495)=3.68, \mathrm{p}=.0003)$.

A finer break down of the n-back task period did not reveal statistically significant differences between the three levels of cognitive demand (i.e., low, moderate, and high). The reference period was significantly different than these three levels of cognitive demand $(F(3,169)=3.18$, $p=0.03)$, a finding in line with the six-minute period analysis. In particular, the participants engaged their signal earlier in the reference period compared to the three periods of added cognitive demand.

\section{Turn Signal Turn-off Time}

Figure 2 presents the turn signal turn-off times with respect to the completion of lane change maneuvers. A positive value indicates that the turn signal was turned off before the completion of the lane change whereas a negative value indicates that the turn signal was turned off after the completion of the lane change. Similar to the turn signal onset time analysis, these data do not include cases when the turn signal was not used during a lane change. A mixed linear model was used for the statistical analysis.

Only the main effect of study period $(F(2,492)=6.44$, $\mathrm{p}=.002$ ) was significant. The mean turn-off times were negative for all three study periods (pre-task: -0.29 sec, nback: -1.20 sec, recovery: $-0.61 \mathrm{sec}$ ), indicating that the participants, on the average, turned off their turn signals after completing the lane change. The participants turned off their turn signals earliest in the pre-task period, followed by the recovery period, and latest in the n-back period (pre-task vs. nback: 1.10 sec, $\mathrm{t}(486)=3.58, \mathrm{p}=.0004$; pre-task vs. recovery: $0.51 \mathrm{sec}, \mathrm{t}(501)=1.97, \mathrm{p}=.049$; recovery vs. n-back: $0.60 \mathrm{sec}$, $t(488)=2.03, p=.04)$. No significant differences were observed between the three levels of cognitive demand (i.e., low, moderate, and high).

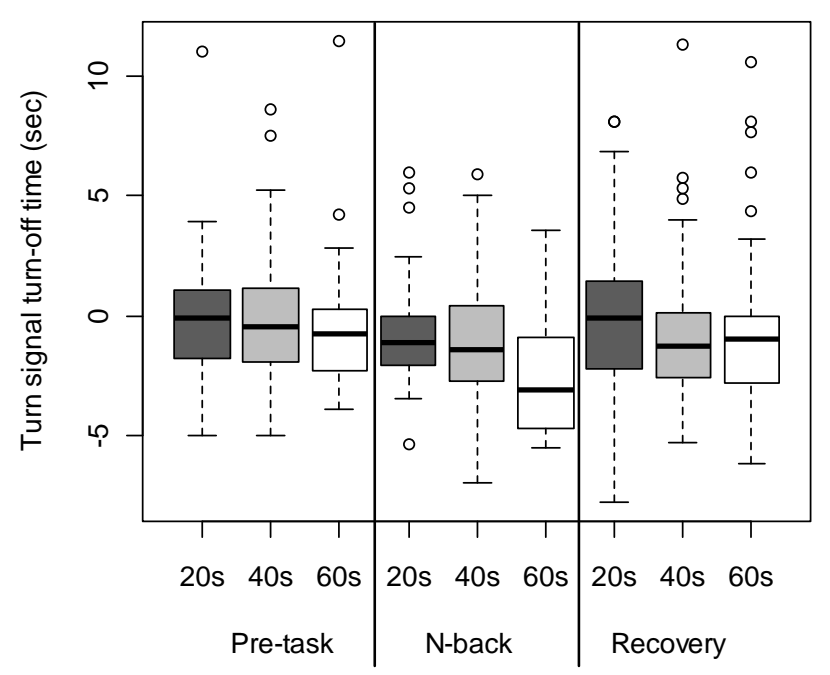

Figure 2. Turn signal turn-off time (sec) for each age and study period (pre-task, n-back, and recovery)

\section{DISCUSSION}

In a field study, we examined turn signal use characteristics of three age groups (20-29, 40-49, and 60-69) under varying levels of cognitive demand as they performed lane changes on the highway. Our initial assessment of lane change behavior in this sample (Lavalliere, et al., 2011), revealed that cognitive demand reduced the likelihood of lane changes for all age groups, suggesting a tendency in drivers of 
all ages to regulate their behavior in a risk reducing direction in response to added cognitive demand. A similar result was not observed for turn signal use rate. Our current findings suggest that added cognitive demand somewhat reduced the likelihood of turn signal use. When participants used their turn signals, added cognitive demand was associated with delayed onset and turn-off of turn signals. In general, it can be argued that the presence of added cognitive load at times led to a neglect of these operational tasks. A delayed turn signal onset arguably increases risk, as other drivers are not provided with an earlier indicator of the imminent lane change. This result is an indication that cognitive demand alters driving behavior in ways that may be detrimental to the safety of road users.

There were no significant differences observed across the three levels of the n-back task. This finding may be due to the relatively limited number of lane changes observed within individual demand levels given their short durations (2 mins).

The presence of an experimenter may have influenced the frequency of turn signal use and the rate in a naturalistic setting might be less than what was observed here. However, we believe that it is reasonable to assume that the same patterns of use in response to increased cognitive demand would likely be observed in a naturalistic setting as well. Further research is needed to validate this assumption.

No major age effects were observed. Turn signal use rate appeared to be largest for older adults (approximately 7\% larger than the other age groups); however, this trend was not statistically significant. Boyce and Geller (2002) observed the opposite trend; they found lower signal use rates for older adults. Their results also were not statistically significant. Given that age is inherently a between-subjects variable, statistical power for this variable may not be high enough to determine a signal-use effect even if the effect exists. It should also be considered that the older subjects in this sample were relatively healthy. Thus, age correlated effects due to health status rather than chronological age would not be expected to influence the results.

Overall, a number of limitations should be considered in the reporting of lane change behaviour in this study. While the sample size is reasonably large for a study of driving behavior, the sample may not be large enough to characterize age effects. As already noted, the short duration cognitive demand periods ( 2 minutes each) limited the number of observed lane changes per level. Additional replications of the demand levels or a larger sample that would increase observations may provide further insight on the effect of incremental increases in demand. The level of resolution at which the initiation of lane changes were coded $(1 \mathrm{~Hz})$ may not provide an optimal level of precision. Future work will need to increase the precision of the detection of initiation points using the full resolution of the $30 \mathrm{fps}$ video. Finally, the lane changes were coded by a single individual and were selectively verified by another individual. Independent coding by multiple individuals, determination of inter-rater reliability to establish consistency to more fully identify any need for adjustments in coding should be undertaken. In spite of these limitations, this study demonstrates a pattern of findings suggestive of the value of undertaking further research in understanding how cognitive demand impacts turn signal usage.

\section{ACKNOWLEDGEMENTS}

We gratefully acknowledge the support of the US Department of Transportation's Region I New England University Transportation Center at MIT and The Santos Family Foundation for the publication of this material and Ford Motor Company for the development of this data source and the instrumented vehicle. Martin Lavallière was supported by a doctoral scholarship and a FRSQ-RQRV International Internship Grant from Fonds de la Recherche en Santé du Québec.

\section{REFERENCES}

Boyce, T. E., \& Geller, E. S. (2002). An instrumented vehicle assessment of problem behavior and driving style: Do younger males really take more risks? Accident Analysis and Prevention, 34(1), 51-64.

Cooper, J. M., Vladisavljevic, I., Medeiros-Ward, N., Martin, P. T., \& Strayer, D. L. (2009). An investigation of driver distraction near the tipping point of traffic flow stability. Human Factors, 51(2), 261-268.

Fitch, G. M., Lee, S. E., Klauer, S., Hankey, J., Sudweeks, J., \& Dingus, T. (2009). Analysis of lane-change crashes and near crashes. (DOT HS 811 147). National Highway Traffic Safety Administration.

Hetrick, S. (1997). Examination of driver lane change behavior and the potential effectiveness of warning onset rules for lane change or "side" crash avoidance systems. Unpublished master's thesis, Virginia Polythecnic Institute and State University, Blacksburg, Virginia.

Kiefer, R. J., \& Hankey, J. M. (2008). Lane change behavior with a side blind zone alert system. Accident Analysis and Prevention, 40, 683-690.

Lavalliere, M., Donmez, B., Reimer, B., Mehler, B., Coughlin, J. F., Klauber, K., et al. (2011). A field study assessing lane changing and lane choice across age and multiple levels of cognitive demand. Proceedings of the Transportation Research Board 90th Annual Meeting. Washington, DC.

Lee, S. E., Olsen, E. C. B., \& Wierwille, W. W. (2004). A comprehensive examination of naturalistic lane changes. (DOT HS 809 702). National Highway Traffic Safety Administration.

Mehler, B., Reimer, B., \& Coughlin, J. F. (2010). Physiological reactivity to graded levels of cognitive workload across three age groups: an on-road evaluation. Proceedings of the Human Factors and Ergonomics Society 54th Annual Meeting. San Francisco, CA.

Mehler, B., Reimer, B., Coughlin, J. F., \& Dusek, J. A. (2009). The impact of incremental increases in cognitive workload on physiological arousal and performance in young adult drivers. Transportation Research Record, 2138, 6-12.

Mehler, B., Reimer, B., \& Dusek, J. (2011). MIT AgeLab delayed digit recall task (n-back), MIT AgeLab White Paper Number 2011-3A. Cambridge, MA: Massachusetts Institute of Technology.

Olsen, E. C. B., Lee, S. E., \& Wierwille, W. W. (2002). Analysis of distribution, frequency, and duration of naturalistic lane changes. Proceedings of the Human Factors and Ergonomics Society 46th Annual Meeting. Baltimore, MD.

Pande, A., \& Abdel-Aty, M. (2006). Assessment of freeway traffic parameters leading to lane-change related collisions. Accident Analysis and Prevention, 38(5), 936-948.

Reimer, B. (2009). Cognitive task complexity and the impact on drivers' visual tunneling. Transportation Research Record, 2138, 13-19.

Reimer, B., Mehler, B., Wang, Y., \& Coughlin, J. F. (2010). The impact of systematic variation of cognitive demand on drivers' visual attention across multiple age groups. Proceedings of the Human Factors and Ergonomics Society 54th Annual Meeting. San Francisco, CA.

Wickens, C. D. (1984). Processing resources and attention. In R. Parasuraman \& R. Davies (Eds.), Varieties of Attention. New York: Academic Press.

Wickens, C. D., \& McCarley, J. S. (2008). Applied Attention Theory. New York, NY: Academic Press.

Zeitlin, L. R. (1993). Subsidiary task measures of driver mental workload: a long-term field study. Transportation Research Record, 1403, 2327. 